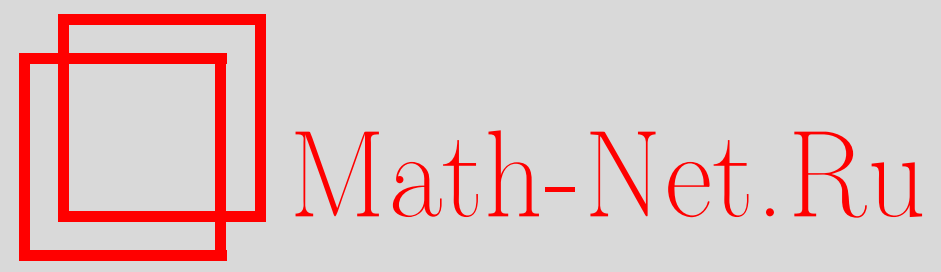

О. И. Шипилов, Эмпирическая функция распределения в эксперименте с биномиальной рандомизацией, Теория вероятн. и ее примен., 1996, том 41, выпуск 2, 310-322

DOI: https://doi.org/10.4213/tvp2936

Использование Общероссийского математического портала Math-Net.Ru подразумевает, что вы прочитали и согласны с пользовательским соглашением http://www . mathnet.ru/rus/agreement

Параметры загрузки:

IP : 35.173 .219 .12

26 апреля 2023 г., 12:56:26

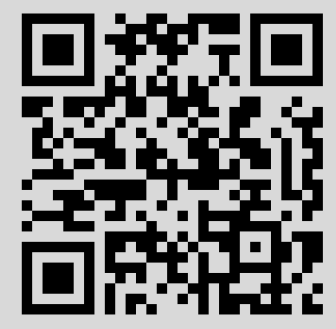




\title{
ЭМПИРИЧЕСКАЯ ФУНКЦИЯ РАСПРЕДЕЛЕНИЯ В ЭКСПЕРИМЕНТЕ С БИНОМИАЛЬНОЙ РАНДОМИЗАПИЕЙ
}

\begin{abstract}
В статье рассматривается эмпирическая функция распределения, порождаемая биномиальным случайным выбором из конечной популяции. Сравнительный биномиально рандомизированный эксперимент представлен как процедура альтернативного биномиального выбора из двух конечных популяций. Вводятся аналоги критериев Колмогорова и Смирнова, основанные на вероятностях, вносимых биномиальным выбором.
\end{abstract}

Ключевье слова и фразы: эмпирическая функция распределения, биномиальный случайный выбор, конечная популяция, рандомизированный эксперимент, критерии Колмогорова и Смирнова.

1. Введение. Одной из актуальных проблем современной математической статистики является разработка методов, позволяющих оценивать свойства конечной совокупности объектов эксперимента по данным о случайным образом (рандомизированно) отобранной части этих объектов. Например, задача оценивания среднего значения количественной характеристики объектов конечной совокупности по среднему значению этой характеристики (признака) в обследованной части этой конечной совокупности оказывается разрешимой, если отбор части, предназначенной к обследованию, осуществляется в строгом соответствии с выбранной процедурой рандомизации, а экспериментальный материал удовлетворяет некоторым условиям регулярности. При этом статистические выводы могут быть сделаны только на основе вероятностей, вносимых в задачу процедурой рандомизации, без обращения к вероятностным моделям для экспериментального материала, если объем обследуемой совокупности достаточно велик. Преимущества и недостатки такого подхода к анализу данных о конечных совокупностях рассмотрены автором в [8]. Наряду с методами статистики конечных совокупностей, направленными на оценивание среднего, развиваются и методы, предназначенные для оценивания функции распределения конечной совокупности с помощью функции распределения обследованной ее части ([1]-[5]), реализован под-

*Центральная аэрологическая обсерватория, ул. Первомайская, 3, 141700, г. Долгопрудный, Московская область, Россия. 
ход с точки зрения статистики конечных совокупностей к оцениванию результатов рандомизированного сравнительного эксперимента ([5]-[8]).

В практике сравнительного рандомизированного эксперимента особое место принадлежит биномиальным планам эксперимента, согласно которым воздействие на некоторый объект назначается или отменяется в соответствии в принятием биномиально распределенной случайной величиной одного из двух значений.

Такие планы эксперимента получили распространение в практике благодаря простоте их реализации и возможности легко управлять объемом эксперимента. Последнее обстоятельство особенно важно при работе с плохо изученным экспериментальным материалом, для которого задача определения объема эксперимента, необходимого для достижения требуемой точности оценок, не обеспечена в должной мере данными предшествуюших опытов. В наиболее общем виде биномиальные планы обследований и экспериментов и соответствующие ощенки для средних конечных совокупностей рассмотрены А. В. Булинским и А. Н. Колмогоровым в [9], [10].

В предлагаемой работе понятие эмпирической функции распределения для наблюдений за конечной совокупностью в ходе рандомизированного эксперимента, введенное в [4], [5] для процедуры рандомизации, соответствующей случайному выбору без возвращения заданного числа объектов из фиксированной конечной совокупности, переносится на рандомизированные обследования и сравнительные эксперименты с биномиальными планами. Введение этого понятия обосновывается с помощью аналога известной теоремы Гливенко-Кантелли [1]; на основе его ра3работаны аналоги критерия Колмогорова и двухвыборочного критерия Смирнова, позволяющие делать выводы о функции распределения признака в обследовании и эксперименте с биномиальной рандомизацией.

В работе приняты следуюшие обозначения: $\mathbf{R}$ - множество всех действительных чисел, $\mathbf{Z}$ - множество всех целых чисел, $\mathbf{Z}_{+}$- множество всех положительных целых чисел; для $z \in \mathbf{R}$ через $\{z\rfloor$ обозначена меньшая часть $z:\lfloor z\rfloor=\max _{y \in \mathbf{Z}}\{y: y \leqslant z\}$. Обозначим $A=\left\{a_{1}, a_{2}, \ldots\right\}$ фиксированную последовательность действительных чисел $a_{k} \in \mathbf{R}, k \in$ $\mathbf{Z}_{+}$, a $B=\left\{b_{1}, b_{2}, \ldots\right\}-$ аналогичным образом введенную последовательность действительных чисел $b_{k}$, которые могут быть не равны $a_{k}$. Пусть $n \in \mathbf{Z}_{+}$, символом $N_{n}$ обозначим множество $\{1,2, \ldots, n\}$. В дальнейшем, при отсутствии специальных указаний, будем считать, что значения всех индексов принадлежат $N_{n}$, а знак $\sum$ без указания множества индексов, по которому проводится суммирование, будет обозначать суммирование по $N_{n}$. Биномиальным планом эксперимента $\varepsilon$ будем называть последовательность независимых в совокупности биномиальных случайных величин $\varepsilon_{k}, k \in \mathbf{Z}_{+}$, принимающих значения 1 и 0 с вероятностью, соответственно, $p_{k}$ и $q_{k}=1-p_{k}$. Обозначение реализации случайной величины будем, когда это необходимо, отличать штрихом от обозначения 
этой случайной величины. Конечную последовательность, образованную первыми $n$ членами $A$ (с сохранением их порядка в $A$ ), обозначим $A_{n}$, аналогичным образом определим $B_{n}=\left\{b_{1}, b_{2}, \ldots, b_{n}\right\}$. Пусть $\theta(x)-$ ступенчатая функция аргумента $x \in \mathbf{R}: \theta(x)=1$ при $x \geqslant 0, \theta(x)=0$ при $x<0$. Величины $p_{k}$ будем полагать удовлетворяюшими условию отделимости:

$$
0<P_{0} \leqslant p_{k} \leqslant P_{1}<1, \quad k \in \mathbf{Z}_{+} \text {. }
$$

2. Эмпирические функции распределения. Пусть $k$-му объекту совокупности из $n$ объектов соответствует значение $b_{k} \in \mathbf{R}$ исследуемой количественной характеристики (признака). Введем, следуя [4], [5], функцию распределения $F_{n}$ множества значений признака $B_{n}$ в совокупности из $n$ объектов:

$$
F_{n}(x)=\frac{1}{n} \sum \theta\left(x-b_{k}\right)
$$

Пусть обследование совокупности $B$ ведется согласно рандомизированному биномиальному плану $\varepsilon$, то есть для $k$-го объекта величина $b_{k}$ измеряется и становится известной в том случае, если случайная величина $\varepsilon_{k}$ приняла значение 1 . В [9] предложены оценки для сумм $\sum b_{k}$ по измеренным согласно плану $\varepsilon$ частям этих сумм:

$$
\widehat{Z}_{0}=\sum \frac{\varepsilon_{k}}{p_{k}} b_{k}, \quad \widehat{Z}_{1}=n\left(\sum \frac{\varepsilon_{k}}{p_{k}}+I_{b}^{n}\right)^{-1}\left(\sum \frac{\varepsilon_{k}}{p_{k}} b_{k}\right),
$$

где $I_{b}^{n}$ - индикатор события $\left\{\sum \varepsilon_{k}^{\prime}=0\right\}$. В соответствии с этим можно предложить следуюшие оценки для $F_{n}(x)$ через измеряемые согласно плану $\varepsilon$ величины $b_{k}$ :

$$
\begin{aligned}
& F_{n}^{*}(x)=\frac{1}{n} \sum \frac{\varepsilon_{k}}{p_{k}} \theta\left(x-b_{k}\right), \\
& \widetilde{F}_{n}(x)=\left(\sum \frac{\varepsilon_{k}}{p_{k}}+I_{b}^{n}\right)^{-1} \sum \frac{\varepsilon_{k}}{p_{k}} \theta\left(x-b_{k}\right),
\end{aligned}
$$

которые могут быть названы эмпирическими функциями распределения, причем $\widetilde{F}_{n}$ будем называть нормированной эмпирической функцией распределения, а $F_{n}^{*}$ - несмещенной эмпирической функцией распределения (нетрудно убедиться, что $\mathbf{E}\left[F_{n}^{*}(x)\right]=F_{n}(x)$. Отметим, что смешение $\widetilde{F}_{n}$ при $p_{k}=p$ не превосходит $(1-p)^{n}$.

С помошью критерия сходимости Колмогорова $[11$, с. 377] легко установить, что при $n \rightarrow \infty$ с вероятностью единища при любом $\boldsymbol{x} \in \mathbf{R}$ выполняется соотношение $\left[F_{n}^{*}(x)-F_{n}(x)\right] \longrightarrow 0$. Однако имеет место и более сильный результат, утверждаюший равномерную сильную состоятельность предложенных оценок и, таким образом, дающий основание называть $\widetilde{F}_{n}$ и $F_{n}^{*}$ эмпирическими функциями распределения. 
Теорема 1. При $n \rightarrow \infty u$ выполнении условия отделимости (1) $c$ вероятностью 1 выполняются соотношения

$$
\begin{aligned}
& \sup _{x \in \mathbf{R}}\left|F_{n}^{*}(x)-F_{n}(x)\right| \longrightarrow 0, \\
& \sup _{x \in \mathbf{R}}\left|\widetilde{F}_{n}(x)-F_{n}(x)\right| \longrightarrow 0 .
\end{aligned}
$$

Доказательство теоремы содержится в работе автора [1], в которой соотношение (3) получено как промежуточный результат при выводе (4).

3. Критерий Колмогорова при биномиальном выборе. Согласно теореме 1 , как $F_{n}^{*}(x)$, так и $\widetilde{F}_{n}(x)$ можно использовать в качестве оценок для функции распределения $F_{n}(x)$. Вопрос о том, какой из этих оценок следует отдать предпочтение, требует изучения распределений этих ощенок, которое приводится ниже в предположении, что $p_{k}=p, 0<$ $p<1$. Анализ этих распределений в значительной степени облегчается тем обстоятельством, что при $p_{k}=$ const, как нетрудно показать, при $t>0$ выполняется неравенство

$$
\begin{aligned}
\mathbf{P} & \left\{\sup _{x \in \mathbf{R}}\left|F_{n}^{*}(x)-F_{n}(x)\right| \leqslant t\right\} \\
& \geqslant \mathbf{P}\left\{\max _{i \in N_{n}}\left|\frac{1}{n} \sum_{k=1}^{i}\left(\frac{\varepsilon_{k}}{p}-1\right)\right|<t\right\}=D_{n}^{p}(t)
\end{aligned}
$$

(равенство в правой части - определение).

Аналогичное неравенство, в правой части которого стоит функция $t$, не зависящая от $B_{n}$, имеет место и для $\mathbf{P}\left\{\sup _{x \in \mathbf{R}}\left|\widetilde{F}_{n}(x)-F_{n}(x)\right|<t\right\}$. Для того, чтобы показать это, расположим величины $b_{k} \in B_{n}$ в ряд по неубыванит. Для совпадаюших между собой значений $b_{i}, b_{k}, \ldots, b_{l}$ сохраним для определенности взаимный порядок их расположения в $B_{n}$. Образованную таким способом последовательность обозначим $\widehat{B}_{n}$. Подмножество $\widehat{B}_{n}$, состоящее из первых $j$ его членов, обозначим $\widehat{B}_{n}(j)$. Нетрудно видеть, что

$$
\begin{aligned}
\sup _{x \in \mathbf{R}}\left|\widetilde{F}_{n}(x)-F_{n}(x)\right| & \\
= & \max _{b_{j} \in B_{n}}\left|\left(\sum \frac{\varepsilon_{k}}{p}+I_{b}^{n}\right)^{-1}\left(\sum \frac{\varepsilon_{k}}{p} \theta\left(b_{j}-b_{k}\right)\right)-\frac{1}{n} \sum \theta\left(b_{j}-b_{k}\right)\right| \\
\leqslant & \max _{j \in N_{n}}\left|\left(\sum \frac{\varepsilon_{k}}{p}+I_{b}^{n}\right)^{-1}\left(\sum \frac{\varepsilon_{k}}{p} I\left[b_{k} \in \widehat{B}_{n}(j)\right]\right)-\frac{1}{n} \sum I\left[b_{k} \in \widehat{B}_{n}(j)\right]\right| \\
= & \left(\sum \frac{\varepsilon_{k}}{p}+I_{b}^{n}\right)^{-1} \\
& \times \max _{j \in N_{n}}\left|\left(\sum \frac{\varepsilon_{k}}{p}-1\right) I\left[b_{k} \in \widehat{B}_{n}(j)\right]-\frac{j}{n} \sum\left(\frac{\varepsilon_{k}}{p}-1\right)\right|
\end{aligned}
$$


Здесь $I\left[b_{k} \in \widehat{B}_{n}(j)\right]=1$, если $b_{k} \in \widehat{B}_{n}(j), I\left[b_{k} \in \widehat{B}_{n}(j)\right]=0$ в противоположном случае.

Переименовав в правой части (6) независимые одинаково распределенные величины $\varepsilon_{k}$ в порядке неубывания соответствующих $b_{k}$ (что не изменит значения вычисляемых вероятностей), из (6) получаем при $t>0$ :

$$
\begin{gathered}
\mathbf{P}\left\{\sup _{x \in \mathbf{R}}\left|\widetilde{F}_{n}(x)-F_{n}(x)\right|<t\right\} \geqslant \mathbf{P}\left\{\max _{j \in N_{n}}\left|\sum_{k=1}^{j}\left(\frac{\varepsilon_{k}}{p}-1\right)-\frac{j}{n} \sum\left(\frac{\varepsilon_{k}}{p}-1\right)\right|\right. \\
\left.<t\left(\sum \frac{\varepsilon_{k}}{p}+I_{b}^{n}\right)\right\}=d_{n}^{p}(t)
\end{gathered}
$$

(равенство в правой части - определение), причем правая часть этого неравенства не зависит от выбора $B_{n}$ и, вследствие этого, от вида $F_{n}(x)$. Заметим, что неравенства (5), (7) обращцаются в равенства в том случае, когда все $b_{k} \in B_{n}$ не равны между собой.

Распределения $F_{n}^{*}(x)$ и $\widetilde{F}_{n}(x)$ при больших $n$ в значительной степени определяются теоремами 2 и 3.

Теорема 2. При $0<p<1, n \rightarrow \infty, t>0$ выполняется соотношеrue

$$
D_{n}^{p}\left(\frac{t}{\sqrt{n}}\right) \rightarrow L\left(t \sqrt{\frac{p}{q}}\right)
$$

гдe

$$
L(x)=\frac{4}{\pi} \sum_{k=0}^{\infty} \frac{(-1)^{k}}{2 k+1} \exp \left\{-\frac{\pi^{2}}{8}(2 k+1) \frac{1}{x^{2}}\right\}
$$

есть функция Реньи.

Д о к а $з$ а т е л в с т в о. Согласно известной теореме о случайном блуждании $[12$, с. 314$]$, при $x>0$

$$
\lim _{n \rightarrow \infty} \mathbf{P}\left\{\max _{j \in N_{n}}\left|\sum_{k=1}^{j}\left(\varepsilon_{k}-p\right)\right| \leqslant x \sqrt{p q n}\right\}=L(x) .
$$

Нетрудно показать, что ряд (8) равномерно сходится в любом интервале $[a, b](a>0)$. Отсюда следует непрерывность $L(x)$ в этом интервале; положив $x=t \sqrt{p / q}$ и заменяя в (9) по непрерывности неравенство на строгое неравенство, получаем утверждение теоремы 2.

Теорема 3. При $0<p<1, n \rightarrow \infty, t>0$ выполняется соотношенuе

$$
d_{n}^{p}\left(\frac{t}{\sqrt{n}}\right) \rightarrow K\left(t \sqrt{\frac{p}{q}}\right)
$$

где $K(x)=\sum_{-\infty}^{\infty}(-1)^{k} e^{-2 k^{2} x^{2}}$ при $x>0$ есть функция Колмогорова. 
Д о к а з а т е л ь с т в о. Выражение (7) для $d_{n}^{p}$ с помощью условных вероятностей может быть при $t>0$ записано в виде

$$
\begin{aligned}
d_{n}^{p}\left(\frac{t}{\sqrt{n}}\right)= & q^{n}\left[1-\theta\left(1-\frac{t}{\sqrt{n}}\right)\right]+p^{n} \\
& +\sum_{m=1}^{n-1} P_{n}(m) \mathbf{P}_{m}\left\{\max _{j \in N_{n}}\left|\sum_{k=1}^{j}\left(\varepsilon_{k}-\frac{m}{n}\right)\right|<t \frac{m}{\sqrt{n}}\right\},
\end{aligned}
$$

где

$$
m=\sum \varepsilon_{k}^{\prime}, \quad P_{n}(m)=\frac{n !}{m !(n-m) !} p^{m} q^{n-m},
$$

$\mathbf{P}_{m}(A)$ - вероятность события $A$ при условии $\sum \varepsilon_{k}^{\prime}=m$. Заметим, что

$$
\mathbf{P}_{m}\left\{\max _{j \in N_{n}}\left|\sum_{k=1}^{j}\left(\varepsilon_{k}-\frac{m}{n}\right)\right|<\frac{a}{n}\right\}=\mathbf{P}\left\{D_{m, n-m}<\frac{a}{m(n-m)}\right\},
$$

где $D_{m, n-m}-$ классическая двухвыборочная статистика Смирнова [13, c. 349], для которой известны как точное распределение, так и асимптотические разложения (см., например, [14] и имеющиеся там дальнейшие ссылки). Таким образом, $d_{n}^{p}(t)$ определяется распределением двухвыборочных статистик Смирнова.

Введем обозначение

$$
\begin{aligned}
\varphi_{n}^{m}(t) & =\mathbf{P}_{m}\left\{\max _{j \in N_{n}}\left|\sum_{k=1}^{j}\left(\varepsilon_{k}-\frac{m}{n}\right)\right|<\frac{t m}{\sqrt{n}}\right\} \\
& =\mathbf{P}\left\{\sqrt{\frac{m(n-m)}{n}} D_{m, n-m}<t \sqrt{\frac{m}{n-m}}\right\} .
\end{aligned}
$$

Известно (см., $[14$, с. 181]), что при $x>0, \min (m, n-m) \rightarrow \infty$ выполняется соотношение

$$
\mathbf{P}\left\{\sqrt{\frac{m(n-m)}{n}} D_{m, n-m}<x\right\} \rightarrow K(x) .
$$

Отсюда следует, что при $m \in s_{n}(\lambda)$, где $0<\lambda<\frac{1}{2}$,

$$
s_{n}(\lambda)=\left\{m: \lambda \leqslant \min \left(\frac{m}{n}, \frac{n-m}{n}\right)\right\}
$$

соотношение

$$
\lim _{n \rightarrow \infty} \mathbf{P}\left\{\sqrt{\frac{m(n-m)}{n}} D_{m, n-m}<x\right\}=K(x)
$$

будет выполняться равномерно по $m \in s_{n}(\lambda)$ при $x>0$. 
Поскольку $K(x)$ при $x>0$ является (как нетрудно показать) непрерывной неубываюпей функцией и $\lim _{x \rightarrow \infty} K(x)=1$, продолжая по непрерывности $K(x)$ равенством $K(x)=0$ при $x \leqslant 0$, получаем функцию распределения $K(x)$, определенную на $\mathbf{R}$. Отсюда, воспроизводя почти дословно доказательство теоремы Пойа $[15$, с. 52], можно показать, что сходимость в (12) равномерна не только по $m \in s_{n}(\lambda)$, но и по R. Вследствие этого для каждого $\delta>0$ найдется $n_{1} \in \mathbf{Z}_{+}$такое, что для всех $n>n_{1}, m \in s_{n}(\lambda), t \in \mathbf{R}$ будут выполняться неравенства

$$
-\delta<\varphi_{n}^{m}(t)-K\left(t \sqrt{\frac{m}{n-m}}\right)<\delta
$$

и вытекающее из них неравенство

$$
-\delta<S_{n}^{p}(t)<\delta
$$

где $S_{n}^{p}(t)=\sum_{m \in s_{n}(\lambda)} P_{n}(m)\left(\varphi_{n}^{m}(t)-K(t \sqrt{m /(n-m)})\right)$.

Таким образом, $\lim _{n \rightarrow \infty} S_{n}^{p}(t)=0$.

Выберем $\lambda \in\left(0, \frac{1}{2}\right)$ таким образом, что $\lambda<\min (p, q=1-p)$, и рассмотрим величину

$$
R_{n}^{p}(t)=T_{n}^{p}(t)-S_{n}^{p}(t)
$$

где

$$
\left.T_{n}^{p}(t)=\sum_{m=1}^{n-1} P_{n} \dot{(} m\right) \varphi_{n}^{m}(t)-\sum_{m=0}^{n} K\left(t \sqrt{\frac{m}{n-m}}\right) P_{n}(m) .
$$

Нетрудно видеть, что

$$
\left|R_{n}^{p}(t)\right| \leqslant 2 \sum_{\{m: m / n<\lambda\}} P_{n}(m)+2 \sum_{\{m:(n-m) / n<\lambda\}} P_{n}(m) .
$$

Согласно закону больших чисел,

$$
\lim _{n \rightarrow \infty} R_{n}^{p}(t)=0
$$

Поскольку $T_{n}^{p}(t)=S_{n}^{p}(t)+R_{n}^{p}(t)$, отсюда следует, что $\lim _{n \rightarrow \infty} T_{n}^{p}(t)=0$.

Заметим, что $P_{n}(m) K\left(t \sqrt{(m / n)(1-m / n)^{-1}}\right)$ образуют последовательность полиномов Бернштейна для непрерывной при $x \in[0,1], t \geqslant 0$ функции $K(t \sqrt{x /(1-x)})$.

Согласно известному доказательству, данному С. Н. Бернштейном для теоремы Вейерштрасса (см., например, $[11$, с. 66]), отсюда следует, что $\lim _{n \rightarrow \infty} \sum_{m=0}^{n} P_{n}(m) K(t \sqrt{m /(n-m)})=K(t \sqrt{p / q})$.

Поскольку $\lim _{n \rightarrow \infty} T_{n}^{p}(t)=0$, а $T_{n}^{p}(t)$ отличается от $d_{n}^{p}(t / \sqrt{n})$ $K(t \sqrt{p / q})$ лишь на величину

$$
q^{n}\left[1-\theta\left(1-\frac{t}{\sqrt{n}}\right)\right]+p^{n}-\left[K\left(t \sqrt{\frac{p}{q}}\right)-\sum_{m=0}^{n} P_{n}(m) K\left(t \sqrt{\frac{m}{n-m}}\right)\right],
$$


стремяшуюся к нулю при $n \rightarrow \infty$, отсюда следует, что

$$
\lim _{n \rightarrow \infty}\left[d_{n}^{p}\left(\frac{t}{\sqrt{n}}\right)-K\left(t \sqrt{\frac{p}{q}}\right)\right]=0 .
$$

Теорема доказана.

Для сравнения качества опенок $F_{n}^{*}(x)$ и $\widetilde{F}_{n}(x)$ нам потребуется следующий результат.

Лемма. $L(x)<K(x)$ прu $x>0$.

Д оказ а те льст во. Поскольку при $x>0$ ряд в правой части (8) есть абсолютно сходящийся ряд с убываюшими по абсолютной величине членами, при $x>0$ выполняется неравенство

$$
L(x)<\frac{4}{\pi} \exp \left\{-\frac{\pi^{2}}{8 x^{2}}\right\}
$$

Наряду с этим, из выражения для $K(x)$, получаемого с помошью преобразования Пуассона [16]:

$$
K(x)=\frac{\sqrt{2 \pi}}{x} \sum_{k=0}^{\infty} \exp \left\{-\cdot \frac{\pi^{2}}{8 x^{2}}(2 k+1)^{2}\right\},
$$

выводим, что

$$
K(x)>\frac{\sqrt{2 \pi}}{x} \exp \left\{-\frac{\pi^{2}}{8 x^{2}}\right\} .
$$

Отсюда следует, что при $x \in\left(0, x_{1}\right)$, где $x_{1}=\pi \sqrt{2 \pi} / 4$, выполняется неравенство

$$
K(x)>L(x)
$$

Альтернативное выражение для $L(x)$ при $x>0[17$, с. 406] можно представить в виде ряда

$$
L(x)=1+\frac{4}{\sqrt{2 \pi}} \sum_{k=1}^{\infty}(-1)^{k} \int_{(2 k-1) x}^{\infty} e^{-t^{2} / 2} d t .
$$

С помощью известного неравенства [18, с. 121]

$$
\frac{1}{x+\sqrt{x^{2}+2}}<e^{x^{2}} \int_{x}^{\infty} e^{-t^{2} / 2} d t \leqslant \frac{1}{x+\sqrt{x^{2}+4 / \pi}}
$$

выполняющегося при $x>0$, нетрудно показать, что ряд (13) абсолютно сходится, а поскольку его члены монотонно убывают по абсолютной величине, при $x>0$ выполняется неравенство

$$
L(x)<1-\frac{4}{\sqrt{2 \pi}}\left(\int_{x}^{\infty} e^{-t^{2} / 2} d t-\int_{3 x}^{\infty} e^{-t^{2} / 2} d t\right) .
$$


Применяя опять (14), устанавливаем, что при $x>0$

$$
L(x)<1-\frac{4}{\sqrt{2 \pi}} \frac{e^{-x^{2} / 2}}{x+\sqrt{x^{2}+4}}+\frac{4 \sqrt{2}}{\pi} \frac{e^{-9 x^{2} / 2}}{3 x+\sqrt{9 x^{2}+8 \pi^{-1}}} .
$$

Из исходного выражения для $K(x)$ имеем

$$
K(x)>1-2 e^{-x^{2} / 2} .
$$

Для доказательства утверждения леммы нам осталось показать, что при $x>x_{1}$

$$
1-2 e^{-x^{2} / 2}>1-\frac{4 \sqrt{2}}{\sqrt{\pi}}\left(\frac{e^{-x^{2} / 2}}{x+\sqrt{x^{2}+4}}-\frac{e^{-9 x^{2} / 2}}{3 x+\sqrt{9 x^{2}+8 \pi^{-1}}}\right),
$$

а это неравенство, как можно показать, следует из легко устанавливаемого при $x>x_{1}$ неравенства

$$
\frac{1}{x+1}>\sqrt{\frac{\pi}{2}} e^{-x^{2} / 2}+e^{-4 x^{2}}
$$

Лемма доказана.

На основании теорем 2 и 3 для функции распределения можно построить доверительные интервалы с заданным асимптотическим доверительным уровнем $1-\alpha$. Пусть $a_{\alpha}(p), b_{\alpha}(p)-$ решения уравнений

$$
K\left(a_{\alpha}(p) \sqrt{\frac{p}{q}}\right)=1-\alpha, \quad L\left(b_{\alpha}(p) \sqrt{\frac{p}{q}}\right)=1-\alpha .
$$

Тогда при $0<\alpha<1$, достаточно большом $n$ и $b_{i} \neq b_{j}(i \neq j)$ будут выполняться приближенно равенства

$$
\begin{aligned}
& \mathbf{P}\left\{\sup _{x \in \mathbf{R}}\left|F_{n}^{*}(x)-F_{n}(x)\right|<\frac{b_{\alpha}(p)}{\sqrt{n}}\right\} \simeq 1-\alpha, \\
& \mathbf{P}\left\{\sup _{x \in \mathbf{R}}\left|\widetilde{F}_{n}(x)-F_{n}(x)\right|<\frac{a_{\alpha}(p)}{\sqrt{n}}\right\} \simeq 1-\alpha .
\end{aligned}
$$

Нетрудно показать, что $L(x)$ и $K(x)$ - неубывающие функции. Отсюда, согласно лемме 2 , следует, что $b_{\alpha}(p)>a_{\alpha}(p)$, то есть оценка $F_{n}^{*}$ при заданном доверительном уровне дает для $F_{n}$ более широкие доверительные интервалы, чем оценка $\widetilde{F}_{n}$. Из таблиц для $K(x)$ и $L(x)[19$, с. 408 , 410] получаем при $p=\frac{1}{2}, \alpha=0,05: b_{0,05}\left(\frac{1}{2}\right)=2,24, a_{0,05}\left(\frac{1}{2}\right)=1,36$. Таким образом, объем обследуемой совокупности $n^{*}$, необходимый для того, чтобы $F_{n^{*}}^{*}$ оценивала $F_{n^{*}}$ с той же асимптотической точностью, с которой $\widetilde{F}_{\tilde{n}}$ оценивает $F_{\widetilde{n}}$, должен быть в $\left(\frac{2,24}{1,36}\right)^{2}=2,7$ раза больше соответствующего объема совокупности $\tilde{n}$. Таков выигрыш от использования нормированной оценки функции распределения вместо несмещенной ее оценки в тех случаях, когда $n^{*}$ и $\tilde{n}$ достаточно велики для того, чтобы 
для оценок можно было использовать предельные теоремы с удовлетворительной точностью. В связи с этим обстоятельством в дальнейшем изложении несмещенная оценка функции распределения не рассматривается. Отметим, что если допустить наличие в данных совпадений, то есть возможность равенства $b_{i}=b_{j}$ при $i \neq j$, то доверительные интервалы, построенные согласно (15), (16), будут накрывать функцию распределения $F_{n}$ с асимптотической доверительной вероятностью, не меньшей, чем $1-\alpha$, обеспечивая тем самым оценку $F_{n}$ с «запасом». Естественной критической областью объема, не большего, чем $\alpha$, для проверки нулевой гипотезы, утверждающей, что функция распределения $B_{n}$ есть $F_{n}(x)$, \& помошью статистики $\sqrt{n} \sup _{x \in \mathbf{R}}\left|\widetilde{F}_{n}(x)-F_{n}(x)\right|$ будет $\left[z_{n}^{p}(\alpha), \infty\right)$, где при заданном $n$ число $z_{n}^{p}(\alpha)$ есть наименьшее число $z$, при котором $d_{n}^{p}(z / \sqrt{n}) \geqslant 1-\alpha$. Согласно теореме 3 отсюда следует, что будет выполняться соотношение

$$
\lim _{n \rightarrow \infty} z_{n}^{p}(\alpha)=a_{\alpha}(p)
$$

Из теоремы 1 заключаем, что критерий, основанный на статистике $\sqrt{n} \sup _{x \in \mathbf{R}}\left|\widetilde{F}_{n}(x)-F_{n}(x)\right|$ и критической области $\left[z_{n}^{p}(\alpha), \infty\right)$, будет сильно состоятелен против последовательности альтернатив, задаваемой функпиями распределения $F_{n}^{1}$, характеризуемой свойством $\sup _{x \in \mathbf{R}} \mid F(x)-$ $F_{n}^{1}(x) \mid>\delta>0$ при $n \in \mathbf{Z}_{+}$. Эт़о свойство предложенного критерия позволяет назвать его аналогом критерия Колмогорова.

4. Сравнительный эксперимент. Аналог двухвыборочного критерия Смирнова. В сравнительном рандомизированном эксперименте проводится сравнение двух последовательностей $A$ и $B$ на основе рандомизированного плана. При биномиальном рандомизированном плане для $k$-го объекта эксперимента с вероятностью $p_{k}$ измеряется величина $b_{k}$ (если $\varepsilon_{k}^{\prime}=1$ ) или с вероятностью $q_{k}=1-p_{k}$ измеряется величина $a_{k}\left(\right.$ если $\left.\varepsilon_{k}^{\prime}=0\right)$. По измеренным в таком эксперименте $n$ величинам требуется вынести суждение о сходстве или различии последовательностей $A_{n}$ и $B_{n}$. Для последовательности $A_{n}$ введем функцию распределения $G_{n}(x)=\sum \theta\left(x-a_{k}\right)$. Оценкой для нее можно взять $\left(\sum\left(1-\varepsilon_{k}\right) / q_{k}+I_{a}^{n}\right)^{-1} \sum\left(\left(1-\varepsilon_{k}\right) / q_{k}\right) \theta\left(x-a_{k}\right)$, где $I_{a}^{n}-$ индикатор события $\left\{\sum\left(1-\varepsilon_{k}^{\prime}\right)=0\right\}$. Нетрудно видеть, что теоремы $1,2,3$ при замене в них $F_{n}, F_{n}^{*}, \widetilde{F}_{n}, \varepsilon_{k}, p_{k}, p, b_{k}$ на, соответственно, $G_{n}, G_{n}^{*}, \widetilde{G}_{n}, 1-\varepsilon_{k}, q_{k}, q, a_{k}$ дословно переносятся на рандомизированное обследование последовательности $A$. Рассмотрим возможность установления в рандомизированном сравнительном эксперименте с $p_{k}=p$ различия между $F_{n}(x)$ и $G_{n}(x)$ по данным наблюдений. Для этой цели примем последовательность гипотез $H_{n}: a_{k}=b_{k}$ и рассмотрим при этой последовательности гипотез распределения аналога двухвыборочной статистики Смирнова. Нетруд- 
но заметить, что при $a_{k}=b_{k}, p_{k}=p$

$$
\begin{gathered}
\mathbf{P}\left\{\sup _{x \in \mathbf{R}}\left|\widetilde{F}_{n}(x)-\widetilde{G}_{n}(x)\right|<t\right\} \geqslant q^{n}[1-\theta(1-t)]+p^{n}[1-\theta(1-t)] \\
+\sum_{m=1}^{n-1} P_{n}(m) \mathbf{P}_{m}\left\{\max _{j \in N_{n}}\left|\sum_{k=1}^{j}\left(\varepsilon_{k}-\frac{m}{n}\right)\right|<t \frac{m(n-m)}{n}\right\}
\end{gathered}
$$

(равенство достигается, если $\left.b_{i} \neq b_{j}(i \neq j)\right)$. Обозначим правую часть этого неравенства $d_{n n}^{p}(t)$. Согласно равенству (11), при любом $\boldsymbol{n}$ величина $d_{n n}^{p}$ может быть вычислена через известные распределения классических двухвыборочных статистик Смирнова $D_{m, n-m}$. Асимптотическое поведение $d_{n n}^{p}$ определяется следующей теоремой.

Теорема 4. При $0<p<1, n \rightarrow \infty, t>0$

$$
d_{n n}^{p}(t) \rightarrow K(t \sqrt{p q})
$$

Д о к а з а т е л ь с т в о. Запишем $d_{n n}^{p}$ с помощью (11) в виде

$$
\begin{aligned}
d_{n n}^{p}\left(\frac{t}{\sqrt{n}}\right)= & q^{n}\left[1-\theta\left(1-\frac{t}{\sqrt{n}}\right)\right]+p^{n}\left[1-\theta\left(1-\frac{t}{\sqrt{n}}\right)\right] \\
& +\sum_{m=1}^{n-1} P_{n}(m) \mathbf{P}\left\{\sqrt{\frac{m(n-m)}{n}} D_{m, n-m}<t \sqrt{\frac{m}{n}\left(1-\frac{m}{n}\right)}\right\} .
\end{aligned}
$$

Введем обозначения:

$$
\begin{aligned}
\psi_{n}^{m}(t) & =\mathbf{P}_{m}\left\{\sup _{j \in N_{n}} \sum_{k=1}^{j}\left(\varepsilon_{k}-\frac{m}{n}\right) \mid<\frac{t}{\sqrt{n}} \frac{m(n-m)}{n}\right\} \\
& =\mathbf{P}\left\{\sqrt{\frac{m(n-m)}{n}} D_{m, n-m}<t \sqrt{\frac{m}{n}\left(1-\frac{m}{n}\right)}\right\}
\end{aligned}
$$

и

$$
C_{n}^{p}(t)=\sum_{m \in s_{n}(\lambda)} P_{n}(m)\left[\psi_{n}^{m}(t)-K\left(t \sqrt{\frac{m}{n}\left(1-\frac{m}{n}\right)}\right)\right]
$$

Здесь, как и ранее, $0<\lambda<\min (p, q), s_{n}(\lambda)=\{m: \lambda<\min (m / n,(n-$ $m) / n)\}$.

Из доказательства теоремы 3 следует, что

$$
\lim _{n \rightarrow \infty} C_{n}^{p}(t)=0 \text {. }
$$

Рассмотрим величину $r_{n}^{p}(t)=\tau_{n}^{p}(t)-C_{n}^{p}(t)$, где

$$
\tau_{n}^{p}(t)=\sum_{m=1}^{n-1} P_{n}(m) \psi_{n}^{m}(t)-\sum_{m=0}^{n} P_{n}(m) K\left(t \sqrt{\frac{m}{n}\left(1-\frac{m}{n}\right)}\right) .
$$


Из очевидного неравенства

$$
\left|r_{n}^{p}(t)\right| \leqslant 2 \sum_{\{m: m / n<\lambda\}} P_{n}(m)+2 \sum_{\{m:(1-m / n)<\lambda\}} P_{n}(m),
$$

согласно закону больших чисел, вытекает соотношение $\lim _{n \rightarrow \infty} r_{n}^{p}(t)=0$ и, следовательно,

$$
\lim _{n \rightarrow \infty} \tau_{n}^{p}(t)=0
$$

Заметим, что при фиксированном $t \geqslant 0$ величины

$$
P_{n}(m) K\left(t \sqrt{\frac{m}{n}\left(1-\frac{m}{n}\right)}\right)
$$

образуют последовательность полиномов Бернштейна для непрерывной при $x \in[0,1]$ функции $K(t \sqrt{x(1-x)})$. Поэтому

$$
\lim _{n \rightarrow \infty} \sum_{m=0}^{n} P_{n}(m) K\left(t \sqrt{\frac{m}{n}\left(1-\frac{m}{n}\right)}\right)=K(t \sqrt{p q}) .
$$

Для завершения доказательства теоремы остается заметить, что $\tau_{n}^{p}(t)$ отличается от $d_{n n}^{p}(t / \sqrt{n})-K(t \sqrt{p q})$ на величину

$$
\begin{aligned}
q^{n}[1 & \left.-\theta\left(1-\frac{t}{\sqrt{n}}\right)\right]+p^{n}\left[1-\theta\left(1-\frac{t}{\sqrt{n}}\right)\right] \\
+ & {\left[K(t \sqrt{p q})-\sum_{m=0}^{n} P_{n}(m) K\left(t \sqrt{\frac{m}{n}\left(1-\frac{m}{n}\right)}\right)\right], }
\end{aligned}
$$

которая стремится к нулю при $\boldsymbol{n} \rightarrow \infty$. Теорема доказана.

Рассмотрим последовательность гипотез $H_{n}$, задаваемую равенствами $a_{k}=b_{k}$, и последовательность альтернатив $K_{n}$, задаваемую неравенствами $\sup _{x \in \mathbf{R}}\left|F_{n}(x)-G_{n}(x)\right| \geqslant \Delta>0$. Определим аналог двухвыборочного критерия Смирнова следующим образом: $H_{n}$ принимается, если $\sup _{x \in \mathbf{R}}\left|\widetilde{F}_{n}(x)-\widetilde{G}_{n}(x)\right|<x_{n}^{p}(\alpha) / \sqrt{n}, H_{n}$ отвергается и принимается $K_{n}$, если $\sup _{x \in \mathbf{R}}\left|\widetilde{F}_{n}(x)-\widetilde{G}_{n}(x)\right| \geqslant x_{n}^{p}(\alpha) / \sqrt{n}$. Здесь $x_{n}^{p}(\alpha)$ - наименьшее $x$, при котором выполняется неравенство $d_{n n}^{p}(x / \sqrt{n}) \geqslant 1-\alpha$ $(1<\alpha<1)$. Нетрудно видеть, что при $H_{n}$ вероятность попадания статистики $\sqrt{n} \sup _{x \in \mathbf{R}}\left|\widetilde{F}_{n}(x)-\widetilde{G}_{n}(x)\right|$ в определенную таким образом критическую область $\left[x_{n}^{p}(\alpha), \infty\right)$ будет не больше $\alpha$. Согласно теореме 4 , $\lim _{n \rightarrow \infty} x_{n}^{p}(\alpha)=c_{\alpha}(p)$, где $c_{\alpha}(p)$ - корень уравнения $K\left(c_{\alpha}(p) \sqrt{p q}\right)=$ 1 - $\alpha$. При альтернативах $K_{n}$, согласно теореме 2 , вероятность выполнения неравенства $\sup _{x \in \mathbf{R}}\left|\widetilde{F}_{n}(x)-\widetilde{G}_{n}(x)\right|>x_{n}^{p}(\alpha) / \sqrt{n}$, начиная с некоторого $n$, становится равной единище. Иначе говоря, данный аналог двухвыборочного критерия Смирнова сильно состоятелен против таких последовательностей альтернатив и способен выявить (при достаточно 
болышом числе рандомизированных опытов) любое регулярное отличие в функциях распределения количественной характеристики объектов эксперимента, могущее возникнуть в результате воздействия на эти объекты.

Автор благодарит рецензента за внимательное прочтение рукописи и за указание направления, в котором следует развивать полученные результаты.

\section{СПИСОК ЛИТЕРАТУРЫ}

1. Шииилов О. И. Аналог теоремы Гливенко-Кантелли при биномиальном выборе из конечной совокупности. - Теория вероятн. и ее примен., 1985, т. XXX, в. 3, c. 574-576.

2. Беляев $Ю$. К., Рыкова J. В. Непараметрический критерий Колмогорова для выборок из конечных совокупностей. - Докл. АН СССР, т. 210, № 6, с. 12611264.

3. Боровских Ю. В. О критерии согласия Колмогорова для выборок из конечных совокупностей. - Докл. АН СССР, 1975, т. 220, № 6, с. 1251-1254.

4. Rosén B. Limit theorems for sampling from finite populations. - Arkiv för Matem., 1964, B. 5, H. 2-3, S. 383-424.

5. Беляев Ю. K. Вероятностные методы выборочного контроля. М.: Наука, 1975, $406 \mathrm{c}$.

6. Булинский.A. В., ШІипилов О. И. О статистике Манна-Уитни в эксперименте с рандомизацией. - Докл. АН СССР, 1983, т. 269, № 4, с. 789-792.

7. Булияский $A$. В., Колжогоров $A$. Н. К оценке эффективности воздействия на облака. - Труды Ин-та прикладной геофизики, 1981, в. 46, с. 69-72.

8. IIипилов $О$. И. Применение статистических методов к оценке эффективности работ по увеличению осадков. - В сб.: Гидрометеорология. Обзорная информация. Сер. метеорология. В. 1. Обнинск: ВНИИГМИ-МЦД, 1983, 24 с.

9. Булинский $A$. B., Колмогоров $A$. $Н$. Јинейные выборочные оценки сумм. - Теория вероятн. и ее примен., 1979, т. XXIV, в. 2, с. 241-251.

10. Булинский $A$. В. Линейные оценки сумм случайных величин. -- Докл. АН СССР; 1981, т. 255 , № 4, c. $784-789$.

11. ІІиряев А. Н. Вероятность. М.: Наука, 1980, 574 с.

12. Спицер Ф. Принципы случайного блуждания. М.: Мир, 1969, 472 с.

13. Хеянекея П. Л., Тортра $A$. Теория вероятностей и некоторые ее приложения. M.: Наука, 1974, 472 c.

14. Королюк B. С., Боровских Ю. В. Аналитические проблемы асимптотики вероятностных распределений. Киев: Наукова думка, 1981, 240 с.

15. Дюее Д. Теоретическая и прикладная статистика. М.: Наука, 1972, 384 с.

16. Смирнов $H$. В. Оценка расхождения между эмпирическими кривыми распределения в двух независимых выборках. - Бюлл. МГУ, 1939, т. 2, в. 2, с. 3-14.

17. Феллер В. Введение в теорию вероятностей и ее приложения. Т. 2. М.: Мир, 1967, $752 \mathrm{c}$.

18. Справочник по специальным функциям. М.: Наука, 1979, 832 с.

19. Большев Л. Н., Смирнов Н. В. Таблицы математической статистики. М.: ВЦ $\mathrm{AH}$ CCCP, $1968,474 \mathrm{c}$. 